\title{
La evolución histórica de los programas musicales en la televisión española: De los programas de divulgación musical a los talent shows
}

institucional.us.es/ambitos/

\author{
Alfonso Blanco Maldonado \\ Universidad Carlos III de Madrid \\ 100349899@alumnos.uc3m.es
}

English Version: The historical evolution of the musical programs in the Spanish televisión: From the programs of musical spreading to the talent shows.

Esta investigación tiene como objetivo evaluar las narrativas y formatos predominantes en los programas musicales en la televisión española desde 1970 hasta hoy. La metodología combina la elaboración de un censo con técnicas cualitativas, a partir de entrevistas a expertos relacionados con el mundo de la música y la televisión. Los resultados apuntan a un predominio del talent show desde el año 2001, que puede ser explicado en clave de la rentabilidad y el interés por contenidos no estrictamente musicales. Esto ha impuesto ciertos patrones de gustos en la población, que han convertido al talent show en el formato musical

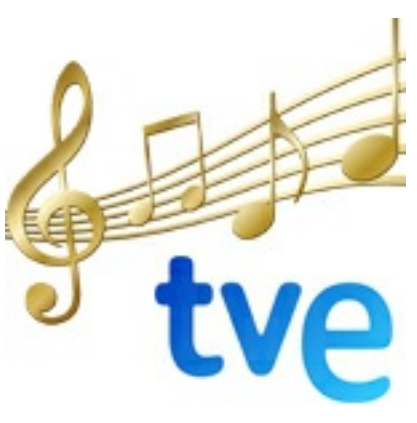
predominante.

\section{Palabras clave}

Talent show, Operación Triunfo, Actualidad musical, Música en directo, Televisión española.

\section{Abstract}

This research tries to evaluate the narratives and predominant formats in the musical programs in the Spanish television from 1970 up to nowadays. The methodology combines the production of a census and qualitative tactics, like interview experts related to the world of the music and the television. The results point at a predominance of the talent show from the year 2001. It is because of profitability and the interest by not strictly musical contents. This has imposed some new interests in the population. Finally, the talent show has been converted into the musical predominant format today.

Keywords

Talent show, Operacion Triunfo, Currence importance, Live music, Spanish television.

\section{INTRODUCCIÓN}

Este trabajo tiene como objetivo analizar la evolución de las narrativas y formatos de los programas musicales que han pasado por la televisión española desde finales de los años 70 (con pioneros como Aplauso o Popgrama) hasta la actualidad, en la que se evidencia un dominio absoluto del talent show desde que en 2001 Operación Triunfo popularizase un nuevo tipo de concurso adaptado a las lógicas de la telerrealidad. El objeto principal es investigar las razones que originaron la evolución de los formatos desde el modelo convencional de programa divulgativo y de actualidad musical hasta su práctica desaparición con la hegemonía del talent show y su búsqueda de rentabilidad a menores costes de producción.

El formato de programa de divulgación musical ha sido habitual en la televisión española desde los comienzos de las emisiones en 1956 (ese año ya estaba la 'Carta de ajuste'), con un momento de máximo apogeo durante 
los años de la Transición a la democracia (década de los 80). Ya en la década de los 90 comenzó a sentirse un cambio sustancial en la programación musical, marcado por la progresiva incorporación de Internet al mercado televisivo así como por la aparición de nuevos formatos como el videoclip, cuya emisión en televisión era excesivamente costosa. Ya entonces programas con un alto coste, música en directo y entrevistas -como fue el caso de Séptimo de Caballería (1998-1999)-, empezaron a registrar índices de audiencia mínimos e hicieron presagiar la tendencia que se impondría desde finales de la década de los 90: una reducción paulatina de los programas de música especializada, que se volvieron residuales, a excepción de emisiones de largo recorrido histórico como Música sí (1997-2004), programa centrado en noticias y actuaciones promocionales en playback. Con la aparición en 2001 de Operación Triunfo se inició una nueva época de auge para el talent, que, enmarcado en las narrativas del del reality show, guió los intereses comerciales de las cadenas privadas en una feroz batalla por conseguir audiencias.

Este estudio intenta llenar el vacío investigador en España acerca de la programación musical de las televisiones generalistas, y, en particular, resolver una pregunta que resuena entre los aficionados a la música: por qué la televisión española ha dejado de contar con programas de divulgación musical. El trabajo se centra, en específico, en un momento fronterizo, acaecido durante la década de los 90 , en el que los programas musicales fueron perdiendo el interés para los programadores, y desencadenaron una mutación radical en las parrillas musicales al privilegiar la fusión de la música con el reality, algo explicable desde una lógica de búsqueda de las audiencias en horario prime time.

\section{METODOLOGÍA}

Para llevar a cabo esta investigación, se ha partido, en primer lugar, de la elaboración de un censo detallado (Cea D’Ancona, 1998) de los programas musicales en Televisión Española desde 1975 hasta la década de los 90 -coincidiendo con la llegada de las televisiones privadas- y se enfatizó en categorías como años de emisión y temáticas. Esta técnica se complementó con la revisión bibliográfica de estudios de referencia en la materia y con una atención especial al estudio de Operación Triunfo (2001) como el programa líder de los talent shows en la televisión española.

Por otro lado, los resultados se triangularon con el uso de métodos cualitativos, en particular, una entrevista en profundidad tanto a ocho diferentes personalidades relacionadas con el mundo de la música y la televisión, con el objetivo de acercar su visión acerca de por qué se produjeron esos cambios significativos en la televisión musical, así como a dos abogados especialistas en reparto de derechos según establece la Ley de Propiedad Intelectual. Se diseñaron dos modelos de entrevistas de tipo semi-estructurado (Vallés, 2003; Taylor y Bogdan, 1992), distinguiendo entre profesionales de los medios y abogados. 
Tabla 1: Relación de personas entrevistadas.

\begin{tabular}{|c|c|c|c|}
\hline ENTREVISTADO & CARGO & $\begin{array}{l}\text { FECHA } \\
\text { ENTREVISTA }\end{array}$ & FORMATO \\
\hline Toñi Prieto & $\begin{array}{l}\text { Directora de programas de } \\
\text { entretenimiento TVE }\end{array}$ & $\begin{array}{l}5 \text { de Abril de } \\
2016\end{array}$ & Correo electrónico \\
\hline $\begin{array}{l}\text { Fernando P. } \\
\text { Paredes }\end{array}$ & $\begin{array}{l}\text { Ex subdirector de } \\
\text { programas musicales TVE } \\
\text { como Música Si y Séptimo } \\
\text { de Caballeria }\end{array}$ & $\begin{array}{l}14 \text { de Mayo de } \\
1016\end{array}$ & Presencial \\
\hline Lara López & $\begin{array}{l}\text { Presentadora y directora de } \\
\text { programas musicales en } \\
\text { TVE y Radio 3, además de } \\
\text { ex directora de Radio } 3\end{array}$ & $\begin{array}{l}17 \text { de Mayo de } \\
2016\end{array}$ & Presencial \\
\hline Javier Hernández & $\begin{array}{l}\text { Director de programas } \\
\text { musicales en Radio } 3\end{array}$ & $\begin{array}{l}17 \text { de Mayo de } \\
2016\end{array}$ & Presencial \\
\hline Eva Tovar & $\begin{array}{l}\text { Directora de programas de } \\
\text { entretenimiento y talent } \\
\text { shows. Actualmente en } \\
\text { 'Got Talent' }\end{array}$ & $\begin{array}{l}30 \text { de Mayo de } \\
2016\end{array}$ & Presencial \\
\hline Esteban Calle & $\begin{array}{l}\text { Coordinador de Marketing } \\
\text { en TVE y productor de } \\
\text { programas musicales. Ex } \\
\text { director de Marketing en } \\
\text { Warner y en EMI }\end{array}$ & $\begin{array}{l}3 \text { de Junio de } \\
2016\end{array}$ & Telefónica \\
\hline Julio Ruiz & $\begin{array}{l}\text { Presentador en Radio } 3 \\
\text { (Premio Ondas) y ex } \\
\text { guionista y subdirector de } \\
\text { programas musicales }\end{array}$ & $\begin{array}{l}17 \text { de Mayo de } \\
2016\end{array}$ & Presencial \\
\hline Aurelio del Portillo & $\begin{array}{l}\text { Realizador, guionista y } \\
\text { presentador en RTVE y } \\
\text { actualmente profesor en } \\
\text { Universidad Rey Juan } \\
\text { Carlos de Madrid }\end{array}$ & $\begin{array}{l}17 \text { de Mayo de } \\
2016\end{array}$ & Correo electrónico \\
\hline Pedro Rivas & $\begin{array}{l}\text { Abogado de Artistas e } \\
\text { Intérpretes. AlE }\end{array}$ & $\begin{array}{l}\text { 11 de Mayo de } \\
2016\end{array}$ & Telefónica \\
\hline Juan Serrada & $\begin{array}{l}\text { Departamento Jurídico } \\
\text { SGAE }\end{array}$ & $\begin{array}{l}27 \text { de Abril de } \\
2016\end{array}$ & Correo electrónico \\
\hline
\end{tabular}

Fuente: Elaboración propia

La entrevista a los expertos en música y televisión intentó abordar fundamentalmente tres dimensiones: en primer lugar, evaluar las razones por las que los talent shows han acabado imponiéndose como el formato musical por excelencia hoy. En segundo lugar, se planteó una batería de preguntas relacionadas con los programas de divulgación y actualidad musical, abordando las razones por las que las que el interés ha declinado y ya no tienen cabida en la televisión actual. Por último, se quiso conocer la opinión de cada uno de 
los expertos en cuanto al futuro de la música en televisión. Por otra parte, con los abogados especialistas se trató directamente cómo la televisión española gestionaba con ellos el pago de los derechos por las actuaciones de los concursantes en los talent, a diferencia de cómo se procedía en los programas de divulgación musical, a fin de conocer las especificidades de ambos tipos de formatos en cuanto al pago de derechos.

Por último, los diferentes testimonios de los informantes clave fueron analizados desde los fundamentos de la teoría fundamentada (Glaser y Strauss, 1967), que inciden en la creación de teorías a posteriori y a partir de las declaraciones recopiladas durante el trabajo de campo. Estas fueron el fundamento de las tres categorías principales que componen el apartado de resultados y que sustentan este estudio.

\section{ORIGEN Y EVOLUCIÓN DE LOS PROGRAMAS DE DIVULGACIÓN Y ACTUALIDAD MUSICAL EN LA TELEVISIÓN ESPAÑOLA}

Quedan lejanos ya aquellos tiempos en los que programas musicales eran, predominantemente, de actuaciones y entrevistas, ofrecían noticias y actualidad musical, y hasta los había transgresores. Desde hace unos años el género reality se ha impuesto en todas sus formas posibles y los espacios que hace unos años eran la vanguardia han desaparecido, siendo el talent show el único musical que prima hoy y que parece mantener el interés de la audiencia. Éste alcanzó su auge en el año 2001 con la llegada de Operación Triunfo, que obtuvo un impactante share del $44,2 \%$ en su primera temporada, en una televisión española en la que los programas musicales ya estaban agonizando. La cuestión es por qué esto ocurrió y a que se debió este cambio en la manera de hacer música en televisión.

De hecho, seguro que muchos recordarán míticos programas que han pasado por la televisión española como Aplauso (1978-1983), total exponente del fenómeno fan y la música comercial, del que tomó el testigo Tocata (1983-1987). Por otro lado, Rockopop (1988-1992), que creó su propia lista de éxitos, y los alejados de las corrientes comerciales, Musical Express (1980-1983) o La edad de Oro (1983-1985). Como señala Borja Terán (2013), hasta 18 programas musicales simultáneos llegó a tener TVE en su parrilla en los años 80 . En los años 90 son menos los programas de música especializada, pero aún nos quedaba Música sí (1997-2004), que con un $22,2 \%$ de share se convirtió en el contenido más visto de la televisión los sábados por la mañana mientras recorría listas de ventas, actuaciones y noticias del sector.

A continuación, se señalan los programas de divulgación y actualidad musical más representativos que han pasado por la televisión española hasta finales de los años 90 y coincidiendo con la llegada de Operación Triunfo. Quedan fuera de esta clasificación los programas de variedades musicales, que incluían actuaciones musicales, números cómicos y de magia (las denominadas "galas"), por no ser considerados objeto de estudio de este trabajo pero que fueron muy habituales en la televisión desde 1957 (Festival Marcon, el primero de ellos). Tampoco se incluyen los talent shows, ya que se les dedicará un apartado especial más adelante.

Se han clasificado estos programas de divulgación y actualidad musical, atendiendo a su contenido, en los siguientes géneros: 1-. Magazine, que incluían tanto entrevistas como reportajes, actuaciones y videoclips; 2-. Actuaciones, o dedicados íntegramente a actuaciones musicales; 3-. Los exclusivamente basados en la emisión de videoclips; 4-. Temáticos, o centrados en el desarrollo de un tema en concreto; 5-. Concursos sobre música, prescindiendo de talent y concursos de cazatalentos; 6-. Y, por último, los que basan su contenido en entrevistas a personajes de la música. 
Tabla 2: Relación de programas musicales desde 1958 hasta 1999.

\begin{tabular}{|c|c|c|}
\hline NOMBRE DEL PROGRAMA & AÑOS EN ANTENA & TIPOLOGIA \\
\hline Teatro Apolo, Teatro Real & 1958 & Música especializada (Lírico) \\
\hline Escala en $\mathrm{Hi}-\mathrm{Fi}$ & 1961-1967 & Actuaciones \\
\hline Tele-Ritmo & 1966-1969 & Actuaciones \\
\hline Último grito & 1966 & Magazine \\
\hline Ritmo 70 & 1970 & Videoclips \\
\hline Diálogos con la música & 1977 & Temático \\
\hline Aplauso & 1978-1983 & Magazine \\
\hline Popgrama & $1977-1981$ & Actualidad \\
\hline Musical Express & $1980-1983$ & Actualidad \\
\hline Música, maestro & 1981 & Especializado (Clásica) \\
\hline Pista libre & $1982-1985$ & Temático \\
\hline ¿Pop qué? & 1984 & Concurso preguntas pop \\
\hline Tocata & $1983-1988$ & Magazine \\
\hline La edad de Oro & 1983-1985 & Magazine \\
\hline Caja de ritmos & 1983 & Actuaciones \\
\hline Autorretrato & 1984 & Entrevistas \\
\hline Jazz entre amigos & 1984-1991 & Música especializada (jazz) \\
\hline Dinamo & 1985-1986 & Temático (deporte asociado a un artista) \\
\hline Metrópolis & 1985 & Temático (Cultura y arte) \\
\hline Auanka buluba balam bambú & 1985-1986 & Magazine \\
\hline La estación de Perpignan & 1987 & Temático \\
\hline ¡Qué noche la de aquel año! & 1987 & Temático (Historia de la música) \\
\hline RockQROP & 1988-1992 & Magazine \\
\hline FM2 & $1988-1990$ & Actualidad \\
\hline Música golfa & 1988 & Actuaciones (Transmisión conciertos) \\
\hline Plastic & 1989-1991 & Magazine \\
\hline Música N.A. & 1989-1991 & Magazine \\
\hline Los conciertos de Radio 3 & 1990-Actualidad & Actuaciones \\
\hline Ponte las pilas & 1990-1992 & Magazine \\
\hline Clip, clap, vídeo & 1991-1992 & Videoclips \\
\hline Area reservada & 1991-1993 & Música especializada (soul y jazz) \\
\hline Música Sí & $1997-2004$ & Magazine \\
\hline Séptimo de Caballería, Séptimo & $1998-1999$ & Entrevistas y actuaciones \\
\hline
\end{tabular}

Fuente: Elaboración propia

\section{HISTORIA DEL TALENT SHOW EN ESPAÑA}

El talent show es una modalidad en la que los concursantes intentan demostrar su talento artístico compitiendo por un premio. Este formato de concurso, aunque ahora ha alcanzado un gran auge, ha existido desde los primeros años de la televisión, cumpliendo la función de crear artistas. Entre estos antecedentes se pueden 
citar como los casos más relevantes a The Original Amateur Hour (1948-1970 y 1992), programa progenitor en el que se han basado todos los talent que conocemos hoy en día, y Star Search (1983-1995) en Estados Unidos.

El precedente de los talent shows se sitúa en la radio, que marcó el inicio de la producción de concursos hasta que la televisión modificó el proceso de producción y adaptó muchos de sus programas. Ya en 1940 un concurso de Radio Barcelona, Los tres cosacos, sentó las bases de concurso en el que se premiaba a un talento, y a principios de los años 50 llegó Conozca usted a sus vecinos (1950-1963), quizá el modelo más conseguido, en el que se descubrieron futuros artistas entonces como Rocío Dúrcal o Ana Belén. Alegría en las ondas permitió el debut en 1959 de un niño llamado José Monge, que pronto sería conocido como Camarón de la Isla. No había cadena que no tuviera su programa de nuevas voces. Radio Nacional de España creó en fecha temprana, 1946, Fiesta en el aire y otros fueron Primer Aplauso (1949-1967), Ruede la bola (1949-1985, todo un récord y plataforma para Raphael o El Fary, Puntadas y Canciones (1958), Varietés Sigma (1959, en la Ser), En pos de la fama (1959), Quiero ser un triunfador (1962), y, limitado a Radio Madrid, Invitación a la fama, y los de Bobby Deglané Cortefiel te brinda la fama (1952) o Vale todo (1960). (Checa, 2010: 4-8).

La importancia que en los primeros días de la televisión tuvo la programación musical está relacionada con la herencia que recibió de la tradición radiofónica: la adaptación de programas típicamente radiofónicos al nuevo medio. (Gómez, 2012: 25). El lenguaje, los contenidos, los profesionales y los planes de producción organizaron una parrilla cuya planificación permitió una mayor y progresiva calidad en la técnica y en el contenido (Moreno, 2014: 39). La televisión española rentabilizó esta fórmula de concurso de talentos con el espacio Hacia la fama (1957-1959). También en 1957, Caras nuevas, con el objetivo de descubrir nuevos rostros que se incorporaran a la plantilla de Televisión Española. Así despega la historia de los talent en la televisión española, que posee una larga y exitosa trayectoria, con ejemplos como el musical Primer éxito (1961), Salto a la fama (1964-65), Canción 71 (1971), La gran ocasión (1972-74) y Gente joven (1974-1987), de donde despegaron las carreras de Isabel Pantoja o María del Monte (Gordillo, 2010: 76, 77). El talent show, tuvo, sin duda, un primer momento de apogeo en la televisión de los años 60 y 70.

Y tras ellos llegó la primera edición de Operación Triunfo en 2001, el momento clave del que trata este estudio, y que cambiaría para siempre el panorama y aportaría una manera singular de mezclar la música, el reality, la emoción, la industria y la construcción de personajes. Operación Triunfo fue el origen en España del fenómeno musical televisivo, puesto que marcó un antes y un después en la manera de hacer televisión en nuestro país. Con su llegada llegó una nueva época de auge para el talent show, que dura hasta hoy, y que conllevó prácticamente la desaparición de los programas musicales tal como los conocíamos hasta entonces. OT tenía su precedente en otros talent de los años 60 y 70 (Pasaporte a Dublín, 1970, total antecedente de OT, pues ya buscaba el candidato a Eurovisión), y que en los años 90 habían sido muy exitosos en la privada (Lluvia de estrellas, 1995-2000), pero en esta ocasión añadía el componente de reality.

\section{CLASIFICACIÓN DEL TALENT SHOW EN LOS GÉNEROS TELEVISIVOS}

Hay autores que apelan a que los géneros televisivos están sometidos al cambio evolutivo del discurso televisivo. La televisión es el medio que más rápidamente evoluciona desde el punto de vista discursivo. Esta transformación afecta a todos los niveles y ha dado lugar, por consiguiente, a la revolución de géneros preexistentes y a la aparición de otros nuevos (Castañares, 1997: 173). Dada la complejidad e hibridación de los formatos, el analista tiene que asumir una tarea que nunca podrá dar por acabada, teniendo en cuenta además que en televisión todo vale con tal de crear siempre programas atractivos que cuenten con el favor de la audiencia. Por tanto, es difícil hacer una teoría de géneros televisivos, teniendo en cuenta que todo cambia y evoluciona constantemente. (Castañares, 1997: 179).

A partir de 1990, el panorama televisivo cambia sustancialmente con el nacimiento de la televisión privada (Antena 3, Tele 5, Canal Plus), que a mediados de los años 80 había comenzado a diversificarse a partir del nacimiento de las televisiones autonómicas y la regulación de la televisión local municipal. Hay que rellenar con más contenido más horas de programación, y surgen nuevos formatos televisivos. A la ficción, entretenimiento e 
información se unen otros contenidos, y aparece el Docudrama. Hay un nuevo protagonista, que es cualquier persona que aparece dentro del discurso televisivo contando su vida. Se empieza a hablar del docudrama como género, a partir de la conjunción de elementos de contenido y fórmulas expresivas. Se trata de un género que acerca a la realidad y que, según establece Inmaculada Gordillo, tiene como manifestaciones el reality show, talk show o talent show. (León, 2009: 25). O también un contenedor dentro del que caben todos estos formatos que apelan a la telerrealidad, y que tienen como punto de inflexión la llegada de Gran Hermano (2000), que consolida la telerrealidad como una dimensión que traspasa las programaciones de la mayoría de canales (Baladrón, 2012: 53).

Por otra parte, está el género concurso, dentro del que se pueden diferenciar entre los quiz shows (si apelan a la inteligencia) o los game shows (a la destreza y espectáculo). Por tanto, según esta teoría, el talent show podría encuadrarse dentro de éstos últimos. O también como una manifestación derivada del docudrama ya que, aparte de tratarse de un concurso musical y de descubrimiento de talentos, acerca la realidad y la convivencia entre un grupo de personas.

Con la llegada de Operación Triunfo, mezcla de concurso musical y reality, se inaugura un modelo de formatofranquicia, que se exporta a más de cincuenta países y que marca las pautas de ese nuevo formato que pervive hasta hoy: formato exportable a otros países, concurso con eliminatorias, gala final como expectativa, jurados profesionales que valoran y el subir a lo más alto a una nueva figura musical. "La fórmula del éxito surgió de la combinación de elementos del reality show, el concurso y el musical. Del concurso incorpora la mecánica de la competición y el premio como recompensa; y del musical extrae el contenido". (Guerrero, 2010: 139). Para algunos, se trata de otro formato encuadrado en el subgénero telebasura por mostrar la esfera íntima y privada de las personas que participan en el mismo e incidir siempre más en los aspectos negativos de los concursantes. Para otros, formaría parte de los programas musicales y educativos, por incentivar el gusto por la música en directo en los más jóvenes. El talent show, por tanto, podría ser un eslabón dentro del docugrama, junto a los realities y todos los formatos de telerrealidad, y también formar parte del género concurso y del formato musical. Las siguientes líneas tienen por objeto desentrañar cuál de ellos es el que cobra más importancia dentro del talent, si la parte estrictamente musical, concurso o la de telerrealidad.

\section{OPERACIÓN TRIUNFO: DIFERENCIAS Y SIMILITUDES CON LOS REALITY GAMES}

Operación Triunfo recogía un conjunto de elementos de otros programas. Su principal referente fue el formato británico Pop Idol (2000) en Reino Unido y estrenado sólo unos meses antes, que recogió la idea básica de Popstars (1999, primer talent show que revolucionó el panorama musical en el nuevo siglo, pero que a España llegó después de OT), e incluyó dos puntos clave de su éxito internacional: participación del público mediante votos para elegir los ganadores (similitud con Gran hermano, 2000) y la incorporación de un jurado. El punto clave era la búsqueda de solistas y no de miembros para formar un grupo musical (diferencia principal con Popstars). (Dafonte, 2011: 53).

Quizás radique en el atractivo del reality gran parte de su éxito, como afirma Wayne Jamison, periodista e investigador del fenómeno OT, en uno de sus libros: "Uno de los méritos que hay que reconocerle a OT es que es especialista en subir a lo más alto a unos jóvenes desconocidos, dejarles probar las mieles del éxito y una fama que desbordaría a más de uno y de dos. El público los ve levantarse, hacer deporte, prepararse los temas" (Jamison, 2007: 56). Un año antes, Gran hermano (2000) había arrasado en España; el público demandaba este formato de reality, y en OT se contaba, además, con unos jóvenes familiares y cercanos que estaban luchando por sus sueños y el camino a la fama, por lo que el atractivo era aún mayor.

Sin renunciar a la vigilancia ni a la mecánica de expulsiones, sometidos al juicio de la audiencia, se trató de convertir la formación en un centro de trabajo en un espectáculo televisivo. El resultado era un formato de telerrealidad que intentaba alejarse de la percepción de programa de telebasura de Gran hermano, que una televisión pública no podía permitirse. Se hacía énfasis en el esfuerzo y trabajo de los concursantes, pasando a un segundo plano las relaciones personales. (León, 2009: 53). Quieren permanecer en el programa el mayor tiempo posible; eso facilita su escalada hacia el éxito. Esta regla del programa no hace más que reformar la 
idea de fama.

Con frecuencia, los realities han estado asociados con el mal gusto. Se les acusa de convertirse en programas tóxicos y sensacionalistas. Estas críticas a Gran hermano se amainaron con la llegada de Operación Triunfo, visto por algunos sectores sociales como un programa limpio, de entretenimiento blanco, sin ningún tipo de malicia, e incluso transmisor de una serie de valores morales como el esfuerzo y la lucha por conseguir una meta. (León, 2009: 87). En OT, se centraban en las galas y preparación de los temas, dejando a un lado los asuntos personales. Se contaba con un objetivo concreto y positivo de la convivencia, solidaridad y compañerismo que supera la rivalidad en el concurso, trabajo en grupo y formación musical. (Dafonte, 211: 62). Muchos vieron en él también una forma de acercar la música al público y, sobre todo en los más pequeños, ya que se convirtió en un programa familiar.

\section{EL ÉXITO DE LOS TALENT SHOWS}

Aunque generen opiniones para todos los gustos, los programas de búsqueda de talento han conseguido imponerse con éxito, han desplazado a los programas musicales como tales, y han generado unas expectativas en la población. En estas emisiones los sentimientos están a flor de piel: Los concursantes ríen, lloran, se entristecen y se emocionan. Congregan tal cantidad de ingredientes procedentes de otros géneros y tal diversidad de contenidos y de aspectos que todos los miembros de la familia telespectadora pueden encontrar algo con lo que emocionarse o identificarse. Y todo ello, con promoción de valores positivos. (Cebrián, 2003: 22). Otro objetivo a seguir por los talent shows es no sólo fomentar el aprendizaje y la superación a nivel vocal y musical de sus aspirantes, sino que también se potencia la convivencia entre sus aspirantes. (León, 2014: 87). La clave está en haber convertido un trabajo académico, la actividad de un centro de formación, en un espectáculo televisivo. Es un elemento motivador para los telespectadores y se expone a la luz pública no sólo lo espectacular de cada gala, sino también el esfuerzo del trabajo para la formación de un artista.

Nunca antes un programa de música había tenido tanto éxito a nivel de audiencia, guste o no el tipo de música, pero bien es cierto que se trata de un formato que da de qué hablar. "Lo verdaderamente importantes es que la televisión ha conseguido que a través de un programa musical millones de telespectadores estén atentos al mismo" (Romero y Degrado, 2005). Los participantes pasaron del anonimato a ser protagonistas, ídolos de masas y personajes creados por la televisión, lo que argumenta la importancia de la selección de los mismos en la clave del éxito del programa. (Moreno, 2014: 40).

Otra de las bazas de los talent shows es que se juega al máximo al suspense para mantener el interés de la audiencia. El formato de concurso le da otro atractivo al proyecto. Se argumentaba que los contenidos musicales no vendían en televisión, pero este proyecto no es sólo un programa musical, sino un concurso con todo el suspense que este género proporciona. (Cebrián, 2003: 60). Hay una evolución de la tensión a medida que avanza el proyecto, generada por las diferentes eliminaciones de concursantes hasta saber quién es el ganador.

La clave del éxito de los talent shows atiende, quizás, a contar con unos concursantes que se convierten en la parte principal del contenido del programa. "Como en toda narración hay trama y suspense (¿quién ganará el concurso?), hay estructuras narrativas (resúmenes, galas), hay personajes (concursantes) y contenidos específicos (entrenamientos, formación, interpretación musical)". (Cebrián, 2003: 95). Y el momento del clímax llega en el momento de dar los resultados sobre quién se salva de los dos propuestos y la votación del jurado; este suspense que se crea con idas continuas a publicidad en el momento de desvelar el misterio, es el momento clave del concurso.

Otro negocio importante, y gran fuente de ingresos que está detrás de todo talent show hoy en día es el de las llamadas y envío de sms. Se incita a la audiencia a que vote para salvar a su concursante preferido (algo que se inició en Gran hermano. Así llega una gran cantidad de dinero al programa, y de la idea de un programa televisivo se pasó a la concepción de una campaña de promoción y venta. (Cebrián, 2003: 24). Desde luego, en los programas de divulgación musical esta suculenta fuente de ingresos no existía aún. 
Lo cierto es que estos programas de talentos, cada vez más espectaculares y fastuosos, han acabado del todo con los programas musicales que conocimos hasta los años 90. David Redondo (2015) ve difícil que a la televisión española vuelvan programas como Aplauso (1978-1983) o Música sí (1997-2002) ya que, desde el cambio grande que se produjo del musical al talent show, la música es más vista en televisión como un elemento para hacer dinero.

Queda mucho por analizar acerca de por qué se produjo este cambio tan grande en la televisión. Tal vez la incursión de las cadenas privadas y la lucha por conseguir el share más alto, o quizás la llegada de Internet y un mundo nuevo de posibilidades donde la música está al alcance de todos. Lo cierto es que el programa musical como formato divulgativo y de actualidad ya apenas tiene espacio en la televisión, mientras que el talent show sigue siendo el único espacio de música en directo por el que las televisiones apuestan.

\section{RESULTADOS DE LAS ENTREVISTAS CON INFORMANTES CLAVE}

\section{1. El Talent show como programa musical predominante hoy}

Si hay un formato que sigue apostando en televisión por la música en directo ese es el talent show. Pero no todos los entrevistados lo consideran programa musical, y las causas que atribuyen a su éxito son distintas.

Precisamente, Toñi Prieto (entrevista personal, 5 de Abril de 2016) piensa que en la novedad y característica diferenciadora es donde está la clave por la que muchos talent shows funcionan. Eso, unido a los concursantes y su cercanía con el público: En Operación Triunfo 1 el casting logró dar con unos concursantes tan naturales que al público le llegaron a resultar muy familiares. En la misma línea se manifiesta Esteban Calle (entrevista personal, 3 de Junio de 2016), que resalta el atractivo del talent en estos concursantes que son artistas noveles, dispuestos a todo, y que no les importa prestarse a una competición con tal de lograr su sueño.

Para algunos entrevistados, no existe relación alguna entre programa musical, como los que conocimos hasta la llegada del siglo XXI, y talent shows. Javier Hernández (entrevista personal, 17 de Mayo de 2016) se siente reacio a este formato, y no lo considera programa musical, sino puramente de entretenimiento. Señala que no es otra cosa que música añadida a un programa de entretenimiento, en el que no importa el talento, sino que cuanto más ridículo y gracioso se es, mejor. También Eva Tovar (entrevista personal, 30 de Mayo de 2016) considera que un talent no es un musical, sino un programa hecho en directo que responde a otros patrones. Tanto ella como Esteban Calle hacen alusión a que hoy funciona lo que se importa, copiando y adaptando. Son formatos ya impuestos que vienen de fuera, repitiendo una fórmula ya de éxito en otros países, y en ellos se le dan al espectador otro tipo de emociones que van más allá de las musicales, remarca Esteban Calle.

Por su parte, Fernando P. Paredes (entrevista personal, 14 de Mayo de 2016) ve claro dónde está el interés del público, poniendo como ejemplo el programa musical de TVE que hoy tiene más audiencia del año: la retransmisión de la gala de Eurovisión. Si analizamos la audiencia de ésta, puede comprobarse que los más altos picos de audiencia están durante las votaciones de cada uno de los países. Las actuaciones musicales importan mucho menos. Esto mismo pasa en los talent shows: el suspense que se crea con las eliminaciones, los juicios de valor a los concursantes y la tensión que se vive cuando se procede al recuento de votos con su posterior resultado. En esto mismo coincide Julio Ruiz (entrevista personal, 17 de Mayo de 2016), que atribuye al éxito del talent show el añadido de convivencia y reality show que tiene éste. Las relaciones personales atraen, además del talento que desarrollan los concursantes. Las eliminatorias, cruces entre concursantes y jurado, y las valoraciones crean mucha expectación en el público.

\subsection{El fin de los programas de actualidad y divulgación musical}

Algunos de los entrevistados achacan el fin de este tipo de programas en televisión a la búsqueda rápida de audiencia. Este es el caso de Fernando P. Paredes, que vio cómo con los talent se aprovechó el momento en el que los realites subían como la espuma tras el filón que supuso Gran hermano (2000), al mismo tiempo que los programas de actuaciones musicales empezaban a decaer. El resultado más ventajoso para la televisión era hacer, entonces, una mezcla, entre música y reality. Para él la principal razón de que no haya programas 
musicales es el share y la audiencia. El público deja de creer en el formato divulgativo. Antes la audiencia no era tan importante; se hacían incluso documentales sin importar esto del share.

Del mismo modo, Julio Ruiz también observa con pena cómo hoy la televisión sólo busca la audiencia. Antes no había tanta lucha por ésta, importaba menos, pero con la llegada de las privadas hay una competencia dura, y la música no es un contenido que vende. Por su parte, Aurelio del Portillo también ve con pena cómo el share hoy importa tanto y ve cómo principal razón la dificultad de venta de estos tipos de programas. Lo que no tiene fácil venta no interesa, y hoy manda el share y las grandes audiencias. Hay que tener millones de espectadores para rentabilizar con publicidad los costes de producción de un contenido audiovisual.

Por otra parte, los hay que consideran que la música siempre ha tenido un hueco difícil en televisión. Lara López, (entrevista personal, 17 de Mayo de 2016), recuerda con nostalgia programas como La Edad de Oro, (1983-1985), Área reservada (1992) o Música N.A. en 1991, que presentó y que considera muy buenos, pero lamenta que este tipo de programa musical haya sido siempre muy escaso en televisión. Otros como Música sí (1997-2004) respondían a un patrón impuesto por las discográficas y no eran más que un escaparate de la industria, hechos para ésta. Para ella, no hay diferencia entre este tipo de programa musical que repasaba listas de ventas, por ejemplo, y los talent que vinieron después. En estos programas la música es sólo vista como un entretenimiento, no como un contenido cultural.

Otra razón que atribuyen otros a la desaparición de los programas y la pérdida de interés de éstos, fue la llegada de Internet. Tanto Toñi Prieto, como Julio Ruiz y Esteban Calle hacen referencia a cómo el consumo ha cambiado, la gente lo ve todo a través de Internet, donde tienen un fácil acceso a los contenidos que le interesan con plataformas como Youtube. Para Esteban Calle, fue ésta una de las plataformas que revolucionó la música y las formas de consumo.

En esto mismo coincide Aurelio del Portillo, que piensa que el público que consume masivamente música y mantiene un elevado interés por grupos, artistas, autores, y por todo su entorno, tiene en Internet una opción más abierta, diversa, de fácil acceso y, en la mayoría de los casos, gratuita. Para Esteban Calle, hoy la información va demasiado deprisa. Gracias a las redes sociales e Internet en general, las noticias no llegan ya a un diario; el público ya se ha informado antes. La televisión no ha podido adaptarse a esa rapidez y, por tanto, un programa hoy de actualidad musical, existiendo Internet, no atraería como lo hacía hace años. Eva Tovar, del mismo modo, remarca la importancia de cómo hoy hay que acceder a Internet y las redes sociales para encontrar ahí contenidos musicales.

Precisamente, Eva Tovar es de las pocas en hacer referencia al maltrato político que sufre la música en España, y que es, sin duda, una razón importante del poco apoyo que tiene ésta en televisión, totalmente distinto al de otros países. No hay que ir mucho más lejos de nuestras fronteras para ver el cambio de visión ante la música. En Cataluña ya el tratamiento y la apuesta es distinta. Barcelona siempre ha tenido una sensibilidad especial con la música y la ven como parte de la cultura, a diferencia de la mayoría del resto de España. También Aurelio del Portillo lamenta el decreciente interés que hay por la cultura hoy en día, y cómo el poder ha fomentado esto.

Si hay algo en lo que coinciden todos los entrevistados, es en que estos programas eran caros. Para todos sin excepción, es el principal motivo por el que las televisiones no los programan. Por ejemplo, Fernando Pérez hace alusión a que la iluminación que necesitan los programas musicales es más espectacular en este tipo de formato y la relación precio-share no va luego unida en este caso, lo que hacía la situación insostenible. La música en directo es muy difícil y cara. Pérez cree que la solución está en pagar a una empresa de sonido un dinero considerable, con el consiguiente disparo del precio. Todo esto con el resultado que todos conocemos: La música no llega a ser rentable en televisión.

Además, señala Pedro Rivas (entrevista personal, 11 de Mayo de 2016), con las actuaciones que se producen en los talent shows no hay que pagar derechos a los intérpretes y ejecutantes, puesto que no existe un fonograma previo. Sin embargo, en los programas de actualidad y divulgación musical sí que había que pagar estos derechos a los artistas, cada vez que se emitía alguna actuación en playback o un videoclip, puesto que en estos casos sí que se basaban en una grabación ya existe. 
La falta de ayuda por parte de las discográficas es otra razón en la que coinciden algunos. Este es el caso de Pérez, que piensa que sí tuvo la televisión interés en esto de volver a hacer programas musicales, pero las discográficas ya no pueden invertir debido a la falta de ingresos por ventas de discos. En esta misma línea está Esteban Calle, que recuerda cómo antes las discográficas iban a las televisiones, ofreciendo a sus artistas, y cómo invertían en traer a los artistas de fuera creándoles un plan de promoción en España. Para las televisiones, la presencia de éstos no les suponía ningún coste. A Eva Tovar le encantaría que hubiese un poquito más de música y artistas emergentes en televisión, aunque sea mediante pequeñas píldoras. Si hubiese detrás una discográfica que pusiese dinero, la televisión apostaría más por ello. Pero, lamentablemente, hoy es muy complicado acuerdo con éstas o con el management, por las razones ya citadas.

\subsection{El futuro de la música en la televisión española}

Lamentablemente, la mayoría de los entrevistados ven un panorama muy negro en la música en televisión. Julio Ruiz no entiende que hoy en día no pueda haber un programa de divulgación en que se pueda dar una noticia musical de realce, y que a veces tengan que ser los informativos los que tengan que dar estas noticias. Mientras no cambie el panorama, la música tendrá un hueco muy difícil en televisión.

Igualmente, Lara López ve que la música, como siga entendiéndose como la cultura aquí, está muerta. La sociedad no está concienciada. $Y$ eso que piensa que hoy contamos con más fórmulas y medios que nunca, y hay muchos profesionales que han hecho lo imposible por innovar. "La asociación música-imagen gusta, pero se necesita una inversión económica y buscar el formato adecuado”. En innovar está la clave también para Esteban Calle. Igual que se ha dado con formatos que posteriormente han sido éxito, en la música habría que pegar el salto a un formato arriesgado, en el que se beneficie al artista. Hay que buscar ideas y formatos buenos para volver a incentivar el interés en las cadenas de televisión. Pero, por desgracia, hoy no hay dinero y no se apuesta lo que se debería.

Toñi Prieto pone como ejemplo el programa Séptimo de Caballería (1998-1999), el cual le resulta curioso que no funcionase como se esperaba, pero no dejaba de ser un programa de culto, del que todo el mundo hablaba como referente de la música. En esto coincide Fernando Pérez, que recuerda la repercusión mediática que tuvo el programa, aunque la audiencia no fuese tan alta. Ahora ya no se realizan acuerdos con discográficas como antes. Sólo tiene hueco la música en las galas de Navidad y/o mezclada con reality (talent) para interesar al público.

En definitiva, todos los entrevistados se muestran pesimistas ante el fututo de la música en televisión, a excepción de Eva Tovar, que cree que la batalla no está perdida. No descarta, y desea, que en el futuro pueda haber programas musicales en televisión con entrevistas y que ofrezcan algo más. Propone como alternativa al coste de los programas, algo tan sencillo como hacer actuaciones en directo a voz y piano, o en formato acústico. No hay que ir siempre a lo espectacular, con tal de que haya música en televisión. Así finaliza Aurelio del Portillo su entrevista, con una frase, cuanto menos, llamativa: "La música no tendrá ningún porvenir. Como no lo tendrá la naturaleza tampoco. No podemos separar unas cosas de otras, creo yo. El problema de raíz es el mismo. Y deberíamos hablar de la lentitud y escasez de ideas con las que están reaccionando los medios tradicionales frente a Internet, que se lo va a tragar todo".

\section{CONCLUSIONES}

Tras los booms de Gran Hermano en el año 2000 y de Operación Triunfo en el 2001, comenzó una nueva etapa para las televisiones, marcada por contenidos más sensacionalistas y la participación activa del espectador, que forma parte del concurso, vota y decide qué concursantes han de llegar hasta el final. Ahora lo que importa es conseguir personajes carismáticos, que cuenten con la simpatía del público y consigan movilizaciones por parte de sus seguidores. Por su parte, la música juega un papel muy secundario, siendo la parte de reality la que le ha ganado la partida.

Los talent forman parte de la nueva televisión que domina el día a hoy, con formatos que han triunfado en otros países como EEUU y que vienen ya impuestos aquí. Son programas que incitan a la expectación y al 
suspense, sobre quién va a ser eliminado o quién va a llegar hasta el final. Se les llama talent shows, pero el talento es lo que menos importa. De hecho, los hay de música, como igualmente de acrobacias, humor o cocina. En definitiva, no son programas musicales; el interés de éstos radica en otros aspectos como la convivencia entre los concursantes y las relaciones o enfrentamientos que puedan surgir entre éstos.

Por otra parte, los programas de actualidad, divulgación y actuaciones musicales, que hace años llegaron a ocupar el prime time incluso, tienen una presencia mínima. Su desplazamiento coincidió con el momento de cambio, a principios del siglo XXI, que vino influenciado por tres factores principales: La caída de la industria musical con la constante bajada en la venta de discos a consecuencia de la piratería, la nueva televisión marcada por los reality y talent shows, y la búsqueda de contenidos musicales en otros lados a través del gigante Internet. Por estos motivos, la música ya había comenzado su declive. En una televisión, en la que el reality empezaba a causar furor, la idea fue entonces mezclar éste con la música. Había que buscar nuevas ideas, ya que en ese momento la música estaba dejando de interesar en televisión, por lo que la primera edición de Operación Triunfo sí que contribuyó a crear en el espectador un gusto por la música, que parecía ya estar aletargado. Pero con todo lo que vino después, importó más el añadido de reality. Los talent son los únicos espacios que ofrecen hoy música en directo y que cuentan con el favor del público, aunque sea ésta justamente hoy la parte del espectáculo que menos interesa.

La música ha ido perdiendo poco a poco interés en los medios, y son muchas las dificultades para promocionar un artista. No se apoya la cultura en este país, y la música ha quedado relegada a un muy segundo plano. Sólo quedan los talent shows, porque forman parte de un espectáculo no solamente musical, sino puramente de entretenimiento y que da audiencia. Pero, la televisión debería educar y formar al espectador para crear en él un interés, gusto y respeto por la música. Del mismo modo, que lo hubo en los años 70 y 80 , hasta que llegaron las privadas y la lucha desencadenada por el share y las audiencias, que sí que ha hecho que se busquen programas de éxito fácil y no interese la cultura porque no da dinero.

Los Conciertos de Radio 3 son una estupenda iniciativa, y es de los pocos espacios hoy en día, aparte de los talent shows, en los que se puede disfrutar de música en directo. El problema está en que, al ser actuaciones calificadas como promocionales de la banda o artista, la televisión no paga cachés, y esto supone un gasto de músicos, alojamientos y desplazamientos que a las discográficas u oficinas de management les cuesta tener que asumir. En una industria en plena reestructuración, tras sufrir una fuerte crisis económica, las discográficas no se quieren permitir estos costes. Esta es, quizás, la más importante causa de que las televisiones no apuesten ya por estos tipos de formatos: No se venden discos, y las discográficas no pueden poner dinero para que sus artistas vayan a televisión. A pesar de todo, ha habido unos buenos intentos muy recientes, como el programa Un lugar llamado mundo (2015), de Javier Limón, o A mi manera (2016.

Muchos son los que atribuyen la llegada de Internet a que la música haya desaparecido en la televisión. Aunque más bien, Internet es una alternativa hoy necesaria para muchos que no encuentran en televisión conciertos ni actuaciones musicales. Por otro lado, las formas de consumo han cambiado y el espectador se siente más partícipe que nunca gracias a los talent shows, pero TVE debería buscar nuevos formatos para indagar e innovar con el panorama musical, del mismo modo que lo hizo a principios de los 2000 con Operación Triunfo y aquel nuevo modelo de televisión, que tanta rentabilidad les dio, pero que no debería haber sido uno de los desencadenantes de la desaparición de los programas musicales que había hasta entonces.

\section{REFERENCIAS BIBLIOGRÁFICAS}

Baladrón, Antonio J. y MUELA, Clara. (2012): “Jóvenes, ídolos mediáticos y nuevos valores”. Revista de Estudios de Juventud, $\mathrm{n}^{\circ} 96$.

Castañares, Wenceslao (1997): “La Televisión y sus géneros: ¿una teoría imposible?”. CIC Cuadernos de Información y Comunicación, 3: 167-181.

Cea D’Ancona, M. A. (1998). Metodología cuantitativa: Estrategias y técnicas de investigación social. Madrid: Síntesis. 
Cebrián, Mariano. (2003): Estrategia multimedia en Operación Triunfo. Madrid: Ciencia 3.

Checa, Antonio (2010): "Los concursos en la radio española. La época dorada. Comunicación y Desarrollo en la era digital". Actas del II Congreso de AE-IC en Málaga, 54 pp. p. 279. http://www.aeic.org/malaga2010/upload/ok/153.pdf [Consulta: 20 de Marzo de 2016].

Dafonte-Gómez, Alberto. (2010): "Evolución de los rasgos culturales del formato televisivo Operación Triunfo en España desde la perspectiva de la identidad de la marca". Revista de Comunicación de la SEECI , nº 25.15 Julio 2011, pp 63-101.

Glaser, B. G. y Strauss, A. L. (1967). The discovery of Grounded Theory: Strategies for qualitative research. Chicago, II: Aldine.

Gómez, Xiana (2012): Contenidos musicales en programación televisiva cultural. Tesis doctoral no publicada. Universidad Autónoma de Barcelona. https://ddd.uab.cat/pub/tesis/2011/hdl_10803_96829/xgd1de1.pdf [Consulta: 20 de Febrero de 2016].

Gordillo, Inmaculada (2010): “Del ‘Responda otra vez' al 'Estás nominado'. Evolución y modelos de los concursos televisivos en España”. Revista Trípodos, n²7. Barcelona, Universitat Ramon Llull, pp. 75-78.

Guerrero, Enrique (2010): El entretenimiento en la televisión española: Historia, industria y mercado. Madrid: Deusto.

Jamison, Wayne (2007): Investigando Operación Triunfo. Madrid: Damar.

León, Bienvenido (2009): Telerrealidad: el mundo tras el cristal. Madrid: Comunicación Social.

León, Yaiza (2014): Los reality games musicales del siglo XXI. Trabajo fin de grado no publicado. Universidad Autónoma de Barcelona. http://ddd.uab.cat/pub/tfg/2014/126061/Yaiza_Leon_de_la_Cruz-_TFG.pdf [Consulta: 7 de Julio de 2016].

Moreno, Julio (2014): “Los concursos en España: Percepción histórica y evolución del género”. Estudios sobre el mensaje periodístico. Vol. 20, 27-42.

Redondo, Javier (2015): “La televisión da la espalda a la música”, en Cadena Ser, 17 de Julio de 2005. http://cadenaser.com/ser/2015/07/17/television/1437142475_056205.html [Consulta: 18 de Enero de 2016].

Romero, Juan Bautista \& Degrado, Ma Dolores (2005): "Programas musicales en la televisión”. Revista Comunicar, $n^{\circ} 25$, p. 368. http://www.revistacomunicar.com/verpdf.php?numero=25\&articulo=25-2005-205 [Consulta: 20 de Febrero de 2016].

Taylor, S. J. y BOGDAN, R. (1992): Introducción a los métodos cualitativos en investigación. La búsqueda de los significados. Barcelona: Paidós.

Valles, M.S. (2003). Técnicas cualitativas de investigación social. Reflexión metodológica y práctica profesional. Madrid: Síntesis.

\section{BREVE SEMBLANZA DEL AUTOR}

Alfonso Blanco Maldonado es Licenciado en Comunicación Audiovisual (2001-2005), por la Universidad de Málaga). Desde el año 2007 desarrolla su trabajo en empresas del sector musical. Este artículo es resultado de una investigación que se realizó para la Universidad Carlos III de Madrid. 
Ámbitos. Revista Internacional de Comunicación, n.35, edición de invierno, 2016-2017. Recibido: 12/10/2016

Aprobado: 16/11/2016 